\title{
Kulturlandschaft gemeinsam verstehen - Praktische Beispiele der Landschaftssozialisation aus dem Schweizer Alpenraum
}

\author{
R. Stotten \\ Universität Innsbruck, Institut für Soziologie, Innsbruck, Austria \\ Correspondence to: R. Stotten (rike.stotten@gmx.ch)
}

Received: 13 July 2012 - Revised: 8 April 2013 - Accepted: 9 April 2013 - Published: 10 July 2013

Zusammenfassung. Within the research field of landscape perception new approaches have been developed and applied on different scales in Switzerland. Agriculture still has a huge impact on the appearance of cultural landscape. The project Kulturlandschaft gemeinsam verstehen investigates the perception among farmers and is focusing on regional differences. Therefore problem-centred interviews have been conducted in two case study areas during an on-site walk with farmers. Regarding the theory of landscape socialisation participative and mediation processes in the realm of landscape values have been considered. The results point out a differentiated perception of cultural landscape within the two case study areas and show that an active inclusion of farmers has a positive impact on the landscape perception.

\section{Einleitung}

Die Kulturlandschaft spiegelt die gesellschaftlichen Strukturen und Veränderungen (Gee, 2010; Hokema, 2009) und stellt sozusagen ,das Archiv der Gesellschaft“ (Knox et al., 2008, 276) dar. Besonders in der Forschung der Landschaftswahrnehmung wurden in den letzten Jahrzehnten von verschiedenen Disziplinen Zugänge zum Verständnis der Landschaft entwickelt (z.B. Backhaus et al., 2008; Ipsen, 2006; Tress und Tress, 2001; Kaplan und Kaplan, 1989). Im Rahmen des Nationalen Forschungsprogramms 48 „Landschaften und Lebensräume der Alpen“ entwickelten Yvan Droz et al. (2006; Droz und Miéville-Ott, 2005) aus einer qualitativen Studie im Bereich der Landwirtschaft in der französischen Schweiz vier Bedeutungen von Landschaft: die erfahrene, die erinnerte, die natürliche und die politische Landschaft. Dabei werden verschiedene Haltungen gegenüber Landschaft über acht verschiedene Werte konstituiert: der produktive, der heilige, der ästhetische, der biologische, der kommerzielle, der identifikatorische, der freizeittechnische und der Habitatwert. Empirische Studien auf europäischer Ebene und in der Schweiz haben die Landschaftswahrnehmung und Landschaftspräferenzen vermehrt von Touristen und Touristinnen (Garrod, 2008; Hunziker et al., 2007), der lokalen Bevölkerung (Hunziker, 2000) oder auf Ebene der Sömmerungsgebiete (Junge et al., 2010) untersucht. Xenia Junge et al. (2011) zeigen in einer quantitativen Studie in der Schweiz Unterschiede und Gemeinsamkeiten der Landschaftswahrnehmung von der allgemeinen Bevölkerung und von Landwirtinnen und Landwirten auf. Demnach zeigen Landwirte und Landwirtinnen, gegenüber der restlichen Bevölkerung, differenzierte Präferenzen in der ästhetischen Wahrnehmung von Landschaft. Nach wie vor hat jedoch die Landwirtschaft durch die Flächennutzung einen grossen Einfluss auf die vorhandene Kulturlandschaft (vlg. auch Junge et al., 2011). Um diese über agrarpolitische Massnahmen zu beeinflussen, stellt sich die Frage, wie Landwirte und Landwirtinnen Kulturlandschaft wahrnehmen. Denn nur wenn die Handlungs- und Wahrnehmungsweisen der landwirtschaftlichen Bevölkerung miteinbezogen werden, kann der Landschaftsschutz nachhaltig umgesetzt werden (Pinto-Correia et al., 2006). Dieser Artikel zeigt beispielhaft anhand von zwei Fallregionen auf, wie Kulturlandschaft von den dort ansässigen Landwirten ${ }^{1}$ wahrgenommen wird.

\footnotetext{
1 Aufgrund der geringen Grösse des Forschungsprojekts beschränkt sich die Untersuchung nur auf die eingetragenen Betriebsleiter bzw. -leiterinnen, die jedoch in diesem Fall alle männlich sind.
} 


\section{Kontext und theoretische Grundlagen}

Aus historischer Sicht kann der Begriff Landschaft bis ins 10. Jahrhundert zurückverfolgt werden. Zur Annäherung an den Begriff hat es schon unzählige unterschiedliche Definitionsversuche aus verschiedenen Zweigen der Wissenschaft gegeben (Ipsen, 2006), wobei ,über den Inhalt des Begriffes doch mehr Übereinstimmung [herrscht], als es zuweilen den Anschein hat." (Meynen und Schmithüsen, 1953, 3, vlg. auch Gailing und Leibenath, 2011). Der Europäischen Landschaftskonvention, die auch von der Schweiz unterschrieben ist, jedoch bislang noch keine formelle Gültigkeit besitzt, liegt folgende Definition zugrunde:

„Landschaft“ [ist] ein Gebiet, wie es vom Menschen wahrgenommen wird, dessen Charakter das Ergebnis der Wirkung und Wechselwirkung von natürlichen und/oder menschlichen Faktoren ist. (Europarat, 2000, Art. 1a)

Die Definitionsgrundlage des Landschaftskonzepts der Schweiz lehnt sich folglich an die oben genannte an:

Landschaft umfasst den gesamten Raum, innerhalb und ausserhalb von Siedlungen. Landschaft ist das Entstandene und Werdende natürlicher Faktoren wie Untergrund, Boden, Wasser, Luft, Licht, Klima, Fauna und Flora in Zusammenspiel mit kulturellen, gesellschaftlichen und wirtschaftlichen Faktoren. (Bundesamt für Umwelt, 2010, 127)

Daraus schliesst sich, dass der physische Raum der Landschaft soziokulturelle Einflüsse mit einschliesst. Der Landschaftsbegriff in dieser Arbeit wird grundlegend nach diesen Definitionen verstanden und gerade die soziokulturellen Aspekte spielen hier eine wichtige Rolle. Weiter kann im semantischen Sinne der Begriff Kulturlandschaft verschieden gedeutet werden. Kultur kann sich auf das gezielte menschliche Handeln beziehen, auf die Bedeutung von gesellschaftlichen Aktivitäten oder auch auf den lateinischen Ursprung des Wortes der Bearbeitung des Bodens (Gailing und Leibenath, 2011; Wöbse, 2002). Gemeinsam ist jedoch den verschiedenen Definitionen von Kulturlandschaft, dass er stets eine Verbindung von Naturraum und menschlicher Aktivität darstellt (Heiland, 2006). Dennoch kommen Ludger Gailing und Markus Leibenath (Gailing und Leibenath, 2011) zu dem Schluss, dass die Differenzierung der Begriffe Landschaft und Kulturlandschaft schwer festzulegen ist. Gleichwohl wird auch die Landschaft im Alpenraum als Kulturlandschaft bezeichnet, das heisst, dass auch sie durch das soziale Handeln des Menschen geprägt und hergestellt wird. Mit dem Wandel der Lebens- und Wirtschaftsweisen der Menschen verändert sich die Kulturlandschaft in einem ständigen Prozess. Besonders geprägt wird die Kulturlandschaft dabei nach wie vor durch die landwirtschaftliche Nutzung (Bätzing, 2003).
Bis zur Modernisierung der Landwirtschaft war schöne Landschaft als Koppelprodukt der landwirtschaftlichen Produktion letztendlich zum Nulltarif für die Gesellschaft erhältlich (Lehmann und Steiger, 2006). Damals stand für die bäuerliche Gesellschaft dabei jedoch nicht die Gestaltung bzw. Pflege der Landschaft im Vordergrund, noch gab es eine bewusste Vorstellung von Landschaft (Krysmanski, 1990; Ritter, 1990). Durch den steigenden Einsatz von Maschinen und weiteren Mitteln der Produktionssteigerung entstand eine auf das Flachland ausgerichtete Landwirtschaft, die keinen umfangreichen Natur- und Landschaftsschutz mehr erbringt - sondern sogar stark zum Rückgang der Biodiversität und Strukturarmut der Landschaft beigetragen hat. Die Landwirtschaft im Berggebiet zur Produktion von Nahrungsmitteln wird dabei unrentabel (Ewald und Klaus, 2009; Umbricht, 2003). Um die traditionelle und naturnahe Kulturlandschaft, wie sie aus der Landnutzung bis Mitte des 20. Jahrhunderts geprägt wurde, zu erhalten, wurden zahlreiche Vorschriften und Anreizsysteme für die Landwirtschaft geschaffen. Damit soll das Marktversagen des Gemeinschaftsgutes Landschaft korrigiert werden, denn Landschaft hat wohl einen Wert, aber keinen Preis auf dem Markt (Lehmann und Steiger, 2006; Weiss, 2006). Mit der eidgenössischen Abstimmung im Juni 1996 hat sich die Schweizer Bevölkerung für eine multifunktionale Landwirtschaft ausgesprochen. Damit wurden neben der Produktion von Nahrungsmitteln, die dezentrale Besiedlung, die Erhaltung der natürlichen Lebensgrundlagen sowie die Pflege der Kulturlandschaft als Ziele der Landwirtschaft in der Bundesverfassung verankert. In der schweizerischen Agrarpolitik sind diese Aufgaben mit dem Ökologischen Leistungsnachweis und den produktungebundenen Subventionen bzw. den Direktzahlungen instrumentalisiert (Norer, 2005).

Während der Beitrag der Bergbauernbetriebe an den verfassungsmässigen Agrarzielen der dezentralen Besiedlung und der Nahrungsmittelversorgung immer kleiner wird, gewinnen die Ziele der Erhaltung der natürlichen Lebensgrundlagen und die Kulturlandschaftspflege an Bedeutung (Bätzing, 2009; Lauber, 2006). Insbesondere der Landwirtschaft im Berggebiet wird die Aufgabe zugesprochen, durch die Pflege der Kulturlandschaft sozialen Nutzen für die Bevölkerung bereitzustellen (Niederer und Anderegg, 1996). Dieser Wandel der Ausrichtung, Bewertung und Entschädigung landwirtschaftlicher Tätigkeiten hat sich jedoch in einem top-down Prozess vollzogen, das heisst er wurde von politischer Ebene implementiert. Innerhalb der landwirtschaftlichen Gemeinschaft wird durch die Erbringung von landschaftspflegerischen Leistungen meist kein inkorporiertes kulturelles Kapital (im Sinne der Kapitallehre nach Bourdieu; vgl. Bourdieu, 2005) erworben und trägt nicht im gleichen Mass zur Kapitalbildung und zur beruflichen Befriedigung bei wie die ursprüngliche, identitätsstiftende Aufgabe der Nahrungsmittelproduktion (Sutherland und Burton, 2011; Stotten et al., 2010; Burton, 2004; Wilson, 2001). Über die Produktion von Nahrungsmitteln, aber auch durch 
weitere landwirtschaftliche Tätigkeiten, wie die Bewirtschaftung von Öko-Ausgleichsflächen, beeinflussen Landwirte in grossem Umfang die vorhandene Kulturlandschaft (Schüpbach et al., 2009). Obwohl Landwirte mit ihrer Tätigkeit einen wesentlichen Teil zur Bereitstellung des öffentlichen Guts Kulturlandschaft leisten, ist wenig darüber bekannt, wie Landwirte und Landwirtinnen Kulturlandschaft wahrnehmen, welches Kulturlandschaftsverständnis sie haben und wie Unterschiede in der Wahrnehmung begründet sind.

Aus konstruktivistischer Perspektive entsteht Landschaft, im Gegensatz zu positivistischen, normativen Ansätzen, durch menschliche Wahrnehmung. Das heisst, durch die Auseinandersetzung des Individuums mit seiner natürlichen Umwelt, die sie hinsichtlich ästhetischer, ökonomischer und anderer Kriterien bewertet und nutzt und somit neu konstruiert (Gailing und Leibenath, 2011). Dieser Prozess vollzieht sich dabei nicht unvoreingenommen, sondern basiert auf bereits gemachten Erfahrungen. In diesem Sinne ist Landschaft „ein Produkt sozial gebildeter Vorstellungen“ (Kühne, 2009; vgl. auch Bourdieu, 1993) und wird von verschiedenen Personen anders wahrgenommen (Lynch, 2007). Olaf Kühne $(2008,2009)$ geht davon aus, dass die Grundlagen zum Lesen und Verstehen der Landschaft während der primären Landschaftssozialisation bereits im Kindesalter vom sozialen Umfeld, wie Familie und Schule, sowie anderen Einflüssen, wie durch Filme, Bücher etc. vermittelt werden. Dabei werden Mechanismen entwickelt, die Landschaft automatisch, im Sinne der Gewohnheit unreflektiert wahrzunehmen. Eine weiterführende, sekundäre Landschaftssozialisation vollzieht sich gemäss Olaf Kühne $(2008,2009)$ nur teilweise, beispielsweise in einem landschaftsbezogenen Studium oder in der Berufsausbildung. Da diese aufbauende Sozialisation in Bezug auf Landschaft nicht alle Personen vollziehen, fehlen im Sinne der Theorie diesen die analytischen Fertigkeiten, die Landschaft bewusst und reflektiert wahrzunehmen und zu beurteilen; also das Expertenwissen. Auch wenn meines Erachtens eine Ebene zwischen der primären und sekundären Landschaftssozialisation vorhanden ist, kann angenommen werden, dass Veränderungen der Landschaft von Laien und Experten anders wahrgenommen und bewertet werden (Tessin, 2008).

Eine umfassende Zusammenstellung der verschiedenen Theorien zur Wahrnehmung von ästhetischen Aspekten der Landschaft hat Steven Bourassa (Bourassa, 1991) zusammengetragen und begründet das ästhetische Empfinden aus der biologischen, kulturellen und individuellen Entwicklung heraus (Bourassa, 1991; vgl. auch Hunziker, 2006). Darüber hinaus wird Landschaft nicht nur visuell wahrgenommen, sondern auch als gelebter Raum des alltäglichen Handelns und Aushandelns wird ihr Bedeutung beigemessen (Gailing und Leibenath, 2011). Für den Landwirt bzw. die Landwirtin stellt das tägliche Handeln die Produktion von Nahrungsmitteln dar; er oder sie selbst nimmt die Kulturlandschaft in erster Linie in Bezug auf seine bzw. ihre Arbeit wahr (Bur- ton, 2012; Schmidt, 2009) und wird als Abbild ihrer eigenen Wirtschaftsweise gesehen: ,good farming is the result of good practice, which again creates good landscapes." (Setten, 2004, 400; vgl. auch Schmidt, 2005; Nassauer und Westmacoot, 1987). Gleiches stellt auch Valérie Miéville-Ott für die Wahrnehmung der Landschaft in der Landwirtschaft in der französischen Schweiz fest: ,[paysage] est beau de qui est bien cultivé.“ (Miéville-Ott, 2001, 100). Ein weiteres Beispiel legt Peter Schallberger (1999) dar: eine Wiese wird dann als schön empfunden, wenn sie eine für den Raum angemessene Bewirtschaftung hat.

Am Institut für Soziokulturelle Entwicklung der Hochschule Luzern - Soziale Arbeit wurde in dem Projekt Kulturlandschaft gemeinsam verstehen von Februar 2011 bis Mai 2012 untersucht, wie Landwirte in zwei verschieden geprägten Regionen der Zentralschweiz Kulturlandschaft wahrnehmen.

Folgende Forschungsfragen werden im Projekt untersucht:

- Wie nehmen Landwirte Kulturlandschaft wahr und welche Faktoren tragen zum Verständnis der Kulturlandschaft bei?

- Wie wirkt sich die Implementierung von partizipativen Verfahren und Vermittlungsprozessen im Hinblick auf das Verständnis von Kulturlandschaft in der Landwirtschaft aus?

\section{Fallgebiete und Methode}

Für die qualitative Untersuchung wurden zwei Fallregionen in der Zentralschweiz ausgewählt: das Urner Oberland und die UNESCO Biosphäre Entlebuch im Kanton Luzern. Die Regionen sind sich bezüglich bestimmter Charakteristika ähnlich: geographisch befinden sich beide Regionen im Alpen- bzw. Voralpengebiet und basieren schwerpunktmässig auf Grünlandwirtschaft. Gleichzeitig unterscheiden sich die gewählten Regionen auch wesentlich: eine Region die im Rahmen der nationalen Parkstrategie (Frey, 2008) unter Schutz steht, und eine Region, die nicht der nationalen Parkstrategie unterliegt. In einem ersten Schritt wurden in beiden Regionen mit Verantwortlichen der Landwirtschaft explorative Gespräche geführt, um zu eruieren in welchem konkreten Gebiet die Interviews mit den Landwirten durchgeführt werden sollen.

Mit der UNESCO Biosphäre Entlebuch (UBE) wurde eine Region gewählt, die unter speziellem Schutz steht. Mit der Annahme der Rothenturm-Initiative wurden 1987 direkt $25 \%$ der Fläche des Entlebuchs unter Schutz für Moore gestellt. Das wurde als Anstoss wahrgenommen, um die Region umfänglich unter Schutz zu stellen und seit 2001 ist das Entlebuch als Biosphärenreservat anerkannt. Diesen Änderungen stand die Bevölkerung des Entlebuchs jedoch zunächst kritisch gegenüber (Backhaus et al., 2008). Untersuchungsparameter in dem Gebiet ist die Gemeinde 


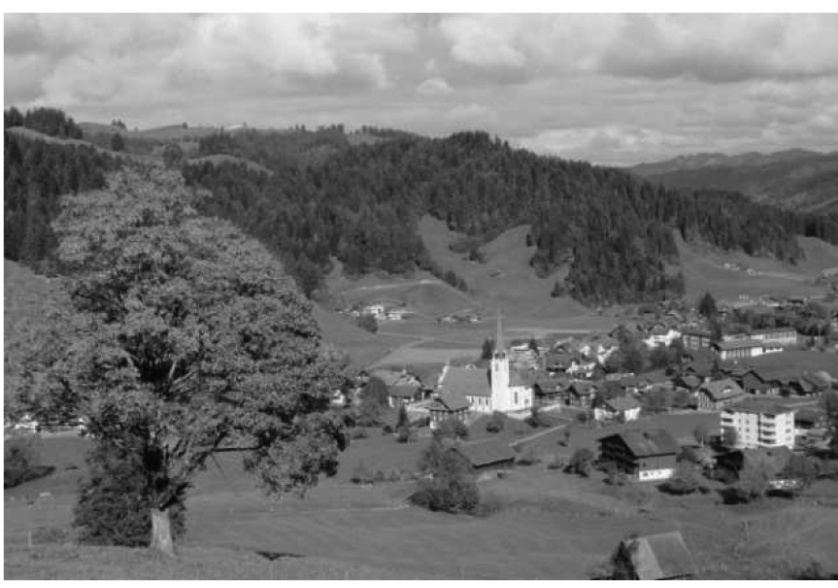

Abbildung 1. Marbach (Quelle: Rike Stotten).

Marbach (vgl. Abb. 1). Mit 20 Stellenprozent ist ein Landwirtschaftsbeauftragter von der Gemeinde angestellt, der sich um die landwirtschaftlichen Belange kümmert und sich für die Vernetzung innerhalb der Region mit der UBE engagiert. Seit 2004 gibt es ein Vernetzungsprojekt im Rahmen der Öko-Qualitätsverordnung unter der Initiative der Gemeinde und Trägerschaft des Landwirtschaftsbeauftragten. An dem Gemeinschaftsprojekt nehmen etwa $70 \%$ der Betriebe teil (Steffen-Odermatt, 2012; Benz et al., 2009). Als aktivierendes Instrument innerhalb der gesamten UBE wirkt das Landwirtschaftsforum. Dieses setzt sich unter der Mitwirkung von Landwirten der Region für eine nachhaltige, regional verankerte Landwirtschaft, sowie Aus- und Weiterbildung in der Landwirtschaft ein und fördert mit diesem partizipativen Ansatz das Zusammengehörigkeitsgefühl innerhalb der Berufsgruppe (UNESCO Biosphäre Entlebuch).

Mit dem Meiental (vgl. Abb. 2) in der Gemeinde Wassen wurde eine zweite Fallregion im Kanton Uri ausgewählt, die nicht im Rahmen der nationalen Parkstrategie geschützt ist. Die Talschaft liegt unterhalb des Sustenpasses und ist durch mehrere bewohnte Weiler besiedelt. Als Reaktion auf die starke Abwanderung wurde 1981 die Vereinigung Pro Meien gegründet, welche der Abwanderung entgegen wirken will (Meier Ziegler und Ziegler, 2013; Diener et al., 2005). Die ansässigen Landwirtinnen und Landwirte sind nicht speziell untereinander organisiert und es gibt keine partizipativen Austauschforen auf regionaler oder kantonaler Ebene.

In beiden Fallregionen wurden die Landwirte direkt von den jeweiligen Kontaktpersonen in den Fallregionen über das Projekt und die geplante Erhebung informiert und zur Teilnahme motiviert. Bedingung für die Auswahl der Landwirte in Marbach zur Teilnahme am Interview war, dass die jeweiligen Landwirte an dem Vernetzungsprojekt der Gemeinde teilnehmen, was auf etwa $70 \%$ der Landwirte in Marbach zutrifft. Weiter sollte ein breites Spektrum von Kriterien wie Alter des Betriebsleiters, Betriebsgrösse und Produktionsform abgedeckt sein. Im Meiental haben sich von acht Landwirten

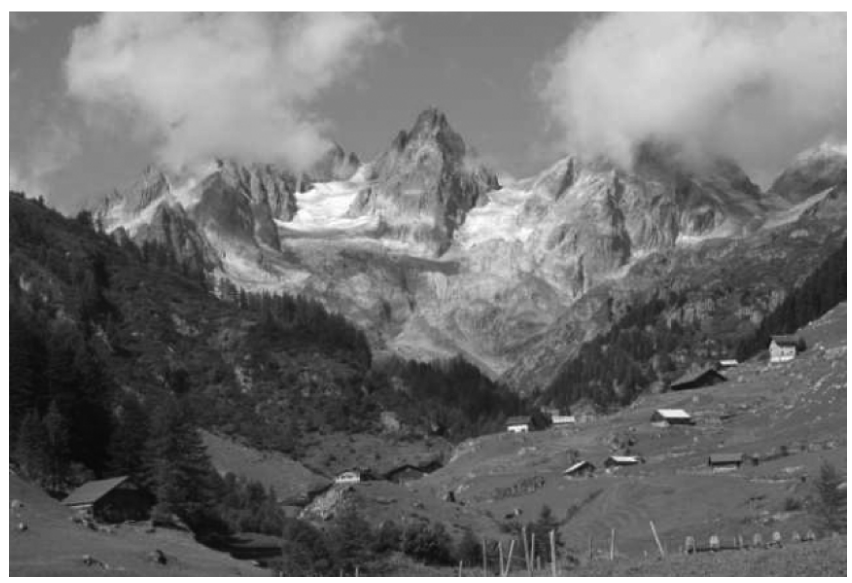

Abbildung 2. Meiental (Quelle: Rike Stotten).

sechs zu einem Interview bereit erklärt. Die einzelnen Landwirte beider Fallgebiete wurden durch die Forschungsperson telefonisch für eine Terminabsprache erstmals kontaktiert.

Für die Erhebung wurde ein Ansatz der qualitativen Sozialforschung gewählt, um mit einem offenen Ansatz neue und tiefere Einblicke der Landschaftswahrnehmung herauszukristallisieren (Lamnek, 2010). Es wurden insgesamt 13 problemzentrierte Leitfadeninterviews mit Landwirten im Alter von 29 und 63 Jahren im Zeitraum von Mitte September bis Mitte Oktober 2011 durchgeführt, sechs davon im Meiental (LW 1-6) und sieben in Marbach (LW 7-13). Als methodisches Vorgehen wurde ein Ansatz der visuellen Soziologie (Pauwels, 2010; Banks, 2007) gewählt, bei welchem die Gespräche mit den Landwirten während eines Spaziergangs auf den jeweiligen Betrieben stattfanden (Burckhardt et al., 2006) und bei denen die Landwirte selber einzelne landschaftliche Elemente auf ihrem Betrieb fotografierten, die sie für wichtig hielten. Dieses Vorgehen wurde gewählt, um die Landwirte im Interview für das Thema Landschaft $\mathrm{zu}$ sensibilisieren und einen erleichterten Einstieg in die Interviewinhalte zu geben (Collier und Collier, 2004; Prosser und Schwartz, 1998). Die Gespräche haben zwischen 40 und 70 Min gedauert und wurden digital aufgezeichnet. Anschliessend wurde ein Interviewprotokoll erstellt und einzelne Passagen des Gesprächs wurden ins Schriftdeutsche transkribiert (Flick, 2007). Die Auswertung der Transkripte erfolgte mit dem computerbasierten Programm ATLAS.ti nach der Methode der qualitativen Inhaltsanalyse von Philipp Mayring (2000, 2010). Dieses Vorgehen stellt eine systematische und regelgeleitete Textanalyse dar, die auf induktiver und deduktiver Kategorienbildung basiert. In der Analyse selbst basiert die Methode auf den einzelnen Schritten der Zusammenfassung, Explikation und Strukturierung. Dabei hat diese Methode nicht den Anspruch, repräsentativ zu sein. Sie zeigt Einstellungen und Meinungen zum Forschungsthema der gewählten Gruppe der Landwirte innerhalb der Untersuchung auf und will menschliches Verhalten erklären (Mayring, 2010). 


\section{Ergebnisse}

Die induktive Kategorienbildung der Inhaltsanalyse wurde anhand des Textmaterials erarbeitet. Im zweiten Schritt der deduktiven Kategorienbildung anhand der zugrunde liegenden Theorien wurden diese Kategorien überarbeitet und aufgrund der Ähnlichkeit an die sechs Dimensionen der Landschaftswahrnehmung (vgl. Abb. 3) nach Backhaus (2008; Backhaus et al., 2007) angepasst. Im Modell gliedern sich diese sechs Dimensionen innerhalb der horizontal und vertikal ausgerichteten Pole Natur - Kultur und Gesellschaft Individuum. Mit dem verwendeten Modell können verschiedene Blickwinkel der Landschaftswahrnehmung sowie Alltagshandlungen die daraus resultieren, differenziert aufgezeigt werden.

\section{1 Ästhetik und Identifikation}

In beiden Fallregionen nehmen die befragten Landwirte Kulturlandschaft sowohl aus ästhetischer Sicht, also mit Bezug auf Merkmale der Schönheit, als auch aus identifikatorischer Sicht aufgrund ihrer beruflichen Tätigkeit als Produzenten von Nahrungsmitteln wahr.

Als ästhetisches Merkmal wird im Meiental von den Landwirten besonders die vorhandene Flora der Kulturlandschaft hervorgehoben (LW2, 4).

Wenn man hier im Frühling so durchgeht, das ist sensationell, das Blumenmeer, das man hier im Meiental hat [...] das ist, also sensationell, und eben wenn das nicht mehr gepflegt würde, sähe man solche Sachen nicht mehr; diese wunderbaren Blumen. (LW 2)

Hier wird die Schönheit der vorhandenen Flora in Zusammenhang gebracht mit Landwirtschaft, die diese Flächen pflegt und erhält. Das Zusammenspiel von vorhandener Kulturlandschaft und Landwirtschaft wird hier zwar geäussert, jedoch nicht bewusst und aktiv dargestellt.

Aus Sichtweise des produzierenden Landwirts nehmen Landwirte im Meiental diejenigen Aspekte der gegebenen Kulturlandschaft als schön wahr, die als gut zu bewirtschaften gelten (LW 1, 3, 4, 6).

Mir gefällt das, weil ich es besser bewirtschaften kann gefällt mir das gute Land besser. (LW 6)

Im Zusammenhang sehe ich die Elemente in der Landschaft eher negativ; das gibt mehr Arbeit, wenn überall wo ich natürlich Haufen und Steine und Ware drin habe, habe ich viel mehr Arbeit, als wenn ich flach durchfahren kann. (LW 3)

Im Einzelnen werden hier Flächen bevorzugt, die flacher sind und keine Bodensteine, Trockenmauern oder andere Hindernisse aufweisen und damit die Bewirtschaftung erleichtern.
Den Zusammenhang der Landwirtschaft mit der vorhandenen Kulturlandschaft bzw. den Beitrag der Landwirtschaft für die Kulturlandschaft sehen die Landwirte in der Offenhaltung der Flächen durch die Nutzung.

In Marbach werden von den Landwirten die vorhandene Kleinstrukturiertheit der Flächen und einzelne Elemente in der Kulturlandschaft als besonders wertvoll und ästhetisch schön thematisiert (LW 7, 8, 9, 10, 11, 13):

Das Kleinräumige, das ist sicher sehr wertvoll. Ist auch schön zum Anschauen, es ist sicher schöner jetzt hier zu wandern oder zu laufen wie vielleicht, wenn man nur irgendein Maisfeld hat, das sieben Kilometer geht und dann kommt wieder ein Weizenfeld, das nachher zehn Kilometer lang ist und die Kleinräumlichkeit, ich denke das ist das, was den Reiz ausmacht. [...] und ich denke, das sind Elemente, die hier das Monotone brechen und das ist wichtig. (LW 9)

Eine Vielfalt von Elementen der Kulturlandschaft wird als wertvoll angesehen und in Vergleich gesetzt mit grossflächiger Landwirtschaft. Auch hier wird der Bezug der Landwirtschaft zum vorhandenen Bild der Kulturlandschaft hergestellt. Weiter werden einzelne Elemente wie Hecken, Bäume und Bäche sogar als natürlich oder zwingend zu einer Landschaft bzw. zum Betrieb als zugehörend wahrgenommen:

Ich habe einfach das Gefühl zu einem Betrieb gehören Obstbäume; das wäre doch hässlich, wenn einfach die Scheune und das Haus allein stehen und kein Baum und Nichts da wäre. (LW 7)

Es wird hier von einem Idealbild des landwirtschaftlichen Betriebs ausgegangen, zu dem auch ein Obstgarten gehört. Auch andere Landwirte stellen den Bezug, zum früheren Aussehen des Betriebes her, welches von ihnen sozusagen als Idealzustand gesehen wird (LW 11, 12), bzw. führen eine Nutzung einzelner Flächen im traditionellen Sinne der Väter bzw. Grossväter fort (LW 7, 9).

Landwirte in Marbach beziehen das vorhandene Bild der Kulturlandschaft auch auf ihre Identifikation mit ihrem Beruf, und nehmen sie aus dieser Perspektive differenziert wahr.

Obwohl es für uns als Bewirtschafter natürlich nicht unbedingt gerade das ist, das am idealsten ist zum Bewirtschaften, aber es ist halt einfach von der Landschaft her auch gegeben, von den Bächen gegeben, von den Wäldern gegeben, [...] aber es ist das, was für mich Kulturlandschaft ausmacht. (LW 9)

Hier wird der Widerspruch zwischen der Kulturlandschaft, die als ästhetisch schön empfunden wird, und einer Kulturlandschaft, die den Idealen der Bewirtschaftung entspricht angedeutet. So entsprechen Flächen, die gut zu bewirtschaften sind, nicht auch zwangsläufig den ästhetischen Ansprüchen der Landwirte. 


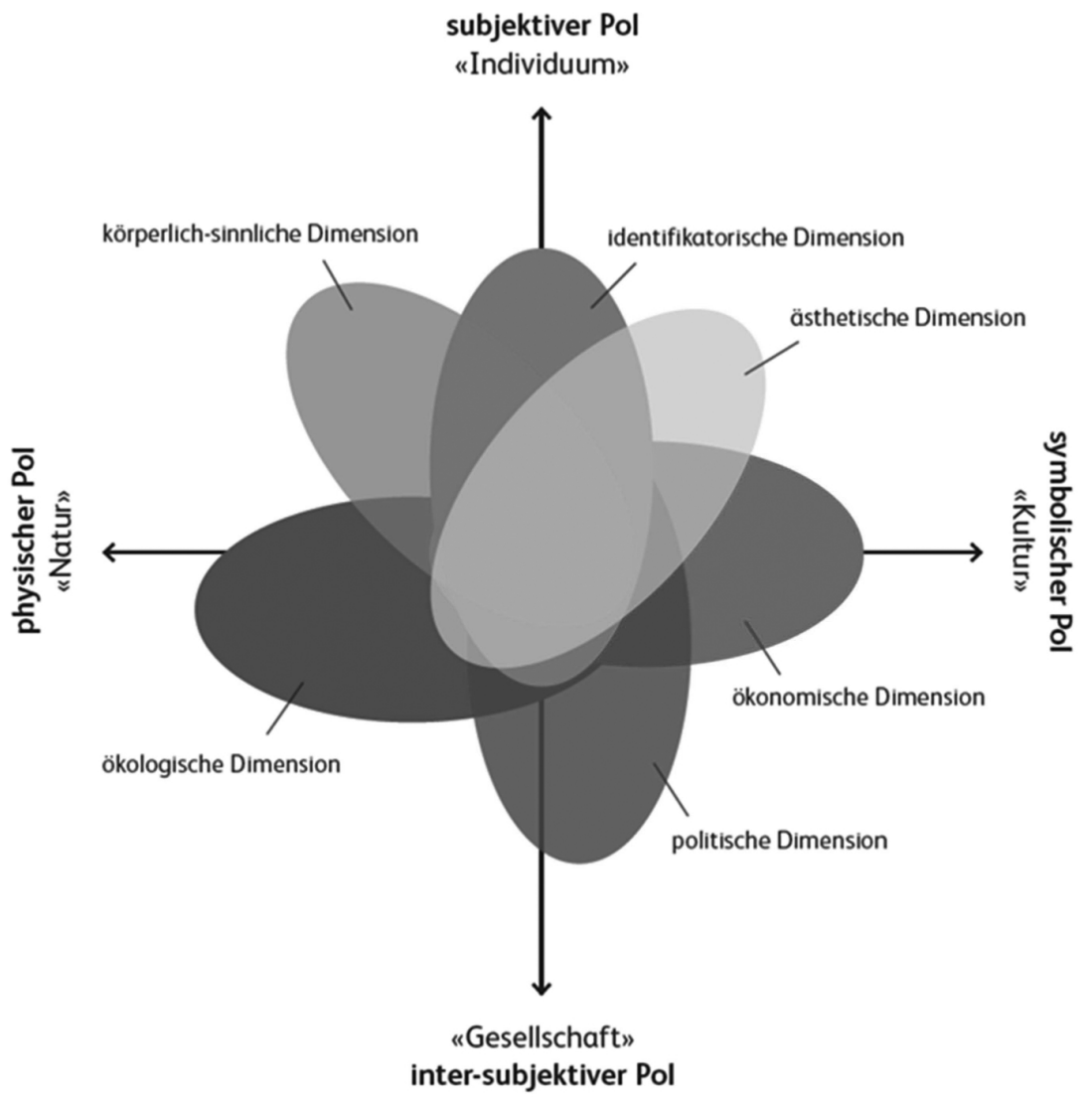

Abbildung 3. Modell der Landschaftswahrnehmung (Quelle: dargestellt nach Backhaus und Stremlow, 2010, 345).

\subsection{Politik und Ökonomie}

Die befragten Landwirte nehmen die Kulturlandschaft aus Perspektive als Betriebsleiter unter Einbezug der vorhandenen agrarpolitischen Rahmenbedingungen wahr. Dazu wird im Meiental der Konflikt des ökonomischen Wachstumsdrucks auf die Landwirtschaftsbetriebe und den dadurch entstehenden Zeitproblem angesprochen. Die Aufgabe des Natur- und Landschaftsschutzes wird deshalb auch kritisch bzw. als problematisch in der Umsetzung angesehen.

Nein man will ja schon, dass Natur erhalten bleibt, aber ich habe Mühe damit. Mit der Landwirtschaft alleine kann man das nicht mehr, das ist unmöglich. Der Landwirtschaftsbetrieb wird eigentlich immer grösser und durch das geht ja Vieles kaputt, weil man da keine Zeit mehr hat. (LW 2)

Auch wird der Verfall bzw. die Aufrechterhaltung einzelner historischer Elemente hinterfragt, jedoch die letztendliche Entscheidung im Umgang damit bei der Gesellschaft gesehen.

Ich weiss nicht, ob das Image von der Landwirtschaft an so einem alten Gebäude hängt. Also, es ist naiv, wir leben einfach nicht mehr in Gott- helfs Zeiten. Ich meine, das was man hier reinsteckt, könnte man anderweitig sinnvoller verwenden. (LW 1)

In Marbach wird der Zusammenhang bzw. der Bezug der Landwirtschaft zur Landschaft als Kompromiss gesehen, der ökonomische Vorteile bietet: Der Mehraufwand wird finanziell abgegolten, stellt aber auch eine Möglichkeit zur vielfältigen und sogar interessanteren Bewirtschaftung dar.

Das sind so Kompromisse, die ich gut eingehen kann; ich profitiere eben wie gesagt von den Beiträgen, aber es macht auch das Bewirtschaften interessant. Es ist halt heute ein Faktor, den man mitplanen und mitberücksichtigen muss. (LW 9)

Durch den Bezug von Abgeltungen entsteht dann ein Pflichtgefühl, für die abgegoltenen Massnahmen, dass man diese Leistung an die Gesellschaft zurückgibt und sichtbar macht. Als grundlegend wird diese Pflicht für ein Tourismusgebiet gesehen.

Weil Bund und Kanton geben ja Geld für die Ökoflächen, [...] da sollte man es auch ein bisschen einhalten und $[\ldots]$ auch etwas zeigen. [...] $\mathrm{Ja}$, und wenn man ehrlich ist, sollte man auch ein 
bisschen beides [die Produktion und den Naturund Landschaftsschutz] haben, in einem Tourismusgebiet sowieso. (LW 8)

Es wird ebenfalls angemerkt, dass heute nicht mehr der finanzielle Anreiz, sondern vielmehr die Überzeugung für den Natur- und Landschaftsschutz motivierend für die Umsetzung solcher Massnahmen ist, was ebenfalls auf die stattgefundene Sensibilisierungsarbeit zurückzuführen ist.

Es war vielleicht im ersten Moment der Anreiz grösser, weil es mit Beiträgen abgegolten worden ist. Aber mittlerweile ist es nicht mehr nur das, weil die Leute hat man jetzt eigentlich ziemlich überzeugen können vom Ganzen. (LW 13)

\section{3 Ökologie}

In Bezug auf die Kulturlandschaft kommen die Landwirte im Meiental nicht auf ökologische Aspekte zu sprechen. In Marbach erwähnen Landwirte lediglich die Notwendigkeit der Kleinräumigkeit als Lebensraum für die Tiere (LW 11, 12).

Keine Erwähnung finden Aspekte in Bezug auf die körperlich-sinnliche Dimension in den Gesprächen mit den Landwirten. Diese Dimension, wie auch die ökologische, positioniert sich im Modell der Landschaftswahrnehmung nach Backhaus (2008; Backhaus et al., 2007) (vgl. Abb. 3) in Richtung Pol „Natur“, als Gegenpol von „Kultur“. Hier wird Natur verstanden als die physischen Grundlagen der Landschaft. Dagegen nehmen Landwirte in dieser Studie Landschaft eher mit Bezug zum Pol Kultur wahr, der auf kulturelle und symbolische Deutungsmuster fokussiert.

\section{Diskussion}

Die im vorherigen Kapitel dargestellten Ergebnisse werden im Folgenden auf die Forschungsfragen der Untersuchung hin diskutiert und überprüft.

\subsection{Wie nehmen Landwirte Kulturlandschaft wahr und welche Faktoren tragen zum Verständnis der Kulturlandschaft bei?}

Im Meiental betonen Landwirte in Bezug auf die Kulturlandschaft zum einen ästhetische Aspekte, wie die vorhandene Flora. Dabei würde es als Verlust empfunden werden, wenn diese Flächen nicht mehr durch die Landwirtschaft gepflegt werden. Auf der anderen Seite wird die vorhandene Kulturlandschaft in Bezug auf die Produktion von Nahrungsmitteln wahrgenommen und Faktoren im Sinne der Produktionsmaximierung tragen zum Verständnis bei. Folgernd werden einzelne Landschaftselemente, wie Bodensteine und Trockenmauern als Hindernis wahrgenommen, da diese den Arbeitsprozess erschweren und mehr Zeitaufwand erfordern.
Im Sinne des inkorporierten kulturellen Kapitals (vgl. Bourdieu, 2005) stehen diese Elemente dem Aufbau von kulturellem Kapital (siehe Abschnitt 2) entgegen. Der grundlegende Beitrag der Landwirtschaft für die vorhandene Kulturlandschaft wird von den befragten Landwirten hauptsächlich in der Offenhaltung der Flächen gesehen. Zu ähnlichen Resultaten kommt auch Marcel Hunziker (2000) in seiner Studie. Nämlich das die Wiederbewaldung nur bis zu einem bestimmten Ausmass als ästhetisch schön empfunden wird, aber darüber hinaus als Verlust von Kulturland.

Landwirte in Marbach nehmen die vorhandene Kulturlandschaft differenziert wahr. Aus ästhetischer Sicht wird die Kleinstrukturiertheit der Flächen betont, die für die Landwirte auch in Zusammenhang mit der Landwirtschaft stehen. So wird der Vergleich, zu Gebieten mit grossflächigem Ackerbau gezogen, welche als nicht beurteilt gesehen werden. Dabei wird von den Landwirten ein Idealbild vom landwirtschaftlichen Betrieb herangezogen; so gehören für einzelne Landwirte bestimmte landschaftliche Elemente grundlegend $\mathrm{zu}$ einem Betrieb, wie der Obstgarten oder extensive Wiesen. Dieses wird mit emotionalen Erinnerungen und Prägungen aus der Vergangenheit begründet und dient als Argument für den weiteren Erhalt dieser Elemente. Diese Auffassungen spiegeln den symbolischen Wert in Richtung des Pols Kultur im Modell der Landschaftswahrnehmung nach Norman Backhaus (Backhaus et al., 2007, 2008) (vgl. Abb. 3) wider. Innerhalb dieser symbolischen Dimension ,,...) wird auch erlernt, was als gut oder schön betrachtet werden kann, ohne dass dies absolute Grössen sind, denn mit sozio-kulturellen Standards ändern sich auch diese Aspekte." (Backhaus, 2010). Mit der primären Landschaftssozialisation nach Olaf Kühne zeigt sich in dieser Studie, dass die Landschaftswahrnehmung während der Sozialisation speziell in der Kindheit auch durch das soziale Umfeld, insbesondere der Familie, geprägt ist, welches Einfluss nimmt auf die Entwicklung verschiedener Haltungen gegenüber Landschaft (vgl. Droz et al., 2006; Droz und Miéville-Ott, 2005) und Bezüge zu bestimmten landschaftlichen Elementen begründet.

In beiden Fallregionen werden von den Landwirten einzelne Elemente der Kulturlandschaft als ästhetisch schön wahrgenommen. Hierbei wurde von den Landwirten in Marbach eine detailliertere Wahrnehmung geäussert und mehrere Elemente einzeln angesprochen. Auch wurde in beiden Fallregionen die Kulturlandschaft aus Perspektive der Identifikation mit dem Beruf im Sinne der Produktion angesprochen. Jedoch wurde von Landwirten in Marbach auch eine Akzeptanz bzw. Befürwortung der Einschränkung der Produktionsmaximierung zur Pflege der Kulturlandschaft dargestellt, die auch als eine Bereicherung für den Berufsalltag gesehen wird. Dagegen zeigen die Landwirte im Meiental eher eine identifikatorische Sichtweise im Sinne der Produktion von Nahrungsmitteln, die mit Unverständnis auf die neuen, multifunktionalen Aufgaben reagiert. 
Im Hinblick auf betriebswirtschaftliche Aspekte sehen sich die Landwirte im Meiental im Konflikt mit der ökonomischen Optimierung durch Flächenzuwachs, vermutlich aus einem Existenzdruck heraus; dadurch werden ihre zeitlichen Ressourcen für die Landschaftspflege eingeschränkt. Auch deshalb weisen Landwirte hier die alleinige Verantwortung für die Pflege der Kulturlandschaft von sich. Dabei erwecken sie jedoch den Eindruck, sie gingen davon aus, die alleinige Verantwortung zu haben - aussen vor bleiben jedoch andere Ebenen, die sich für die Pflege der Kulturlandschaft einsetzen. In einzelnen Aussagen wird der Sinn der Erhaltung und Pflege der Kulturlandschaft in Frage gestellt und dabei von den befragten Landwirten als nicht mehr zeitgemäss beurteilt. Es drückt sich aus, dass hier Landwirte eher nicht den Wert von historischen Elementen der traditionellen Landwirtschaft anerkennen und dieses vermutlich einwirkt auf die Umsetzung der agrarpolitischen Ziele. Dieses kann in Bezug gesetzt werden zu der oben aufgeführten Annahme (vgl. Lehmann und Steiger, 2006), dass Landschaft keinen Preis auf dem Markt hat. Obwohl das Markversagen mit der Agrarpolitik korrigiert werden soll, stellt das Gemeinschaftsgut Kulturlandschaft für die Landwirte jedoch eher einen geringen Wert dar.

Von den Landwirten in Marbach wird der Zusammenhang zwischen betriebswirtschaftlichen Aspekten und den agrarpolitischen Auflagen als Mittelweg gesehen, der dabei aber auch den Beruf des Landwirts aus ihrer Sicht vielfältiger und interessanter macht. Dabei sehen sie sich in der Verpflichtung, der Gesellschaft für den Bezug von Abgeltungen über den Bund, auch etwas zurück zugeben zu müssen und auch zu wollen. Als Leistungen dafür werden der Naturund Landschaftsschutz gesehen. Es wird eingeräumt, dass zu Beginn der Umsetzung von Natur- und Landschaftsschutz die finanziellen Anreize ausschlaggebend für ihre Beteiligung daran waren. Jedoch nehmen die Landwirte selbst auch einen Wandel der Einstellung zum Natur- und Landschaftsschutz, sowie den Wandel des Berufsbildes Landwirt wahr. Dieses widerspricht den zuvor dargestellten Annahmen, dass durch die Erbringung von landschaftspflegerischen Leistungen kein kulturelles Kapital erzeugt wird (Sutherland und Burton, 2011; Stotten et al., 2010; Burton, 2004; Wilson, 2001). Landwirte aus Marbach zeigen hier Ansätze für die Entwicklung von kulturellem Kapital durch die Erbringung von Leistungen für den Natur- und Landschaftsschutz.

\subsection{Wie wirkt sich die Implementierung von partizipa- tiven Verfahren und Vermittlungsprozessen in Hin- blick auf das Verständnis von Kulturlandschaft in der Landwirtschaft aus?}

Die Ergebnisse der Interviews mit den Landwirten aus Marbach zeigen, dass sich die Einstellung der Landwirte in Bezug auf Natur- und Landschaftsschutz geändert hat. Sie bestätigen, dass sich der Einbezug in die UBE und die damit einhergehende Sensibilisierungsarbeit sowie der Parti- zipationsprozess prägend auf die Wahrnehmung der Kulturlandschaft bei den Landwirten auswirken. Landwirte in Marbach zeigen eine vielschichtige Wahrnehmung der Kulturlandschaft aus verschiedenen Blickwinkeln; so wird von den befragten Landwirten nicht nur die Perspektive des Landwirts als Nahrungsmittelproduzent geäussert, sondern die Landschaft auch losgelöst von der landwirtschaftlichen Tätigkeit im Sinne der Produktion betrachtet. Miteinbezogen in ihre Überlegungen werden die Bedürfnisse anderer Nutzergruppen, wie diejenigen der Touristen oder teilweise auch der Fauna. Dagegen äussern Landwirte im Meiental hauptsächlich die Wahrnehmung der Kulturlandschaft aus Perspektive des Landwirts als Nahrungsmittelproduzent, also mit Bezug zur landwirtschaftlichen Arbeit im Sinne der Produktion. Gleichzeitig wird das Thema des Natur- und Landschaftsschutzes im Meiental weder unter den Landwirten selbst, noch in einem anderen, interaktiven Gefäss ausgetauscht.

Olaf Kühne $(2008,2009)$ geht innerhalb seiner Theorie in der sekundären Landschaftssozialisation von der Aneignung von analytischen Fähigkeiten zur reflektierten und bewussten Wahrnehmung der Landschaft in einem landschaftsbezogenen Studium aus. Die Ergebnisse der vorliegenden Untersuchung jedoch deuten darauf hin, dass auch partizipative Sensibilisierungsarbeit den Anstoss zur Auseinandersetzung mit den vielseitigen Herangehensweisen an die Kulturlandschaft geben kann. Demnach kann die Landschaftswahrnehmung der Gewohnheit im Sinne der primären Landschaftssozialisation erweitert werden und sich auch ohne spezielle, landschaftsbezogene Aus- oder Weiterbildung hin zu einem reflektierten und analytischen Umgang mit Landschaft entwickeln.

\section{Schlussfolgerungen}

Die Studie zeigt auf, dass die Landschaftswahrnehmung der untersuchten Landwirte eher von kulturellen Aspekten als von physischen Grundlagen (vgl. Modell der Landschaftswahrnehmung Backhaus, 2010) geprägt ist. Die zuvor diskutierten Ergebnisse verdeutlichen, dass die Landwirte für den Verständnisaufbau und die Wahrnehmung ihrer Funktionen neben der Produktion von Nahrungsmitteln als Dienstleister für die Gesellschaft zur Kulturlandschaftspflege mittels partizipativer Ansätze sensibilisiert werden sollten (vlg. Miéville-Ott und Droz, 2010; Schenk et al., 2007), da dadurch die Landschaftswahrnehmung in Richtung der sekundären Landschaftssozialisation hin zu einer analytischen und reflektierten Wahrnehmung der Landschaft weiterentwickelt werden kann. Dieser Aspekt sollte auch vermehrt in Aus- und Weiterbildung für Landwirtinnen und Landwirte integriert werden. Aus den Ergebnissen wird weiter angenommen, dass sich partizipative Verfahren und Vermittlungsprozesse positiv auf die Selbstwahrnehmung des Landwirts (vgl. Stotten et al., 2010) als multifunktionalem 
Dienstleister auswirken. Hier bedarf es tiefergreifender Untersuchungen zu der Frage, inwieweit sich partizipative Verfahren und Vermittlungsprozesse langfristig auf die Bildung von inkorporiertem kulturellem Kapital bei der Erbringung von landschaftspflegerischen Leistungen auswirken. Ferner sollte über solche Prozesse auch das Gemeinschaftsdenken der Landwirte gefördert werden, da Landwirte heute nicht mehr nur Konkurrenten bei der Nahrungsmittelproduktion sind, sondern darüber hinaus auch Kooperationspartner in der Kulturlandschaftspflege ihrer Region.

Diese Studie lässt vermuten, dass neben dem inkorporierten kulturellen Kapital (vgl. Sutherland und Burton, 2011; Stotten et al., 2010; Burton, 2004) auch der Habitus (vgl. Bourdieu, 1979) als strukturierendes Element für die Landschaftswahrnehmung eine wesentliche Rolle spielt. Das heisst, dass der Habitus einerseits die verinnerlichten Strukturen der Landschaftswahrnehmung aus der primären Landschaftssozialisation darstellt, und andererseits auch die Grundlage für soziale Gewohnheiten der Landschaftswahrnehmung bezeichnet. Da sich der Habitus, also die Gewohnheiten der Landschaftswahrnehmung, je nach soziokulturellen Standards verschiedener sozialer Gruppen, differenziert, stellt sich für die Zukunft die Frage, inwieweit Unterschiede in der habituell geprägten Landschaftswahrnehmung regional erkennbar sind. Bisherige Forschung im Bereich der Landschaftswahrnehmung (wie eingangs dargestellt) berücksichtigen diesen Ansatz nicht, jedoch besteht hier, auch in Bezug auf aktuelle Diskussionen um die kulturelle Dimension Nachhaltiger Entwicklung (European Cooperation in Science and Technology), weiterer Forschungsbedarf.

Acknowledgements. Ich danke den Bauern, die sich für die Mitwirkung an dieser Studie die Zeit genommen haben. Weiter bedanke ich mich bei Rebekka Ehret und den anonymen Reviewern für die kritische Durchsicht des Manuskripts und die gehaltvollen Anregungen.

Edited by: B. Korf

Reviewed by: O. Kühne and two anonymous referees

\section{Literatur}

Backhaus, N.: Macht und Kraft der Bilder: ein (preisgekröntes) Beispiel transdisziplinärer Forschung, in: Le défi de l'inter- et transdisciplinarité, Herausgeber: Darbellay, F. und Paulsen, T., Presses polytechniques et universitaires romandes, Lausanne, 239260, 2008.

Backhaus, N.: Landschaften wahrnehmen und nachhaltig entwickeln : ein transdisziplinäres Modell für Forschung und Praxis, Geogr. Helv., 65, 48-58, doi:10.5194/gh-65-48-2010, 2010.

Backhaus, N., Reichler, C., und Stremlow, M.: Alpenlandschaften: Von der Vorstellung zur Handlung thematische Synthese zum Forschungsschwerpunkt I "Prozesse der Wahrnehmung und Darstellung von Landschaften und Lebensräumen der Alpen"; Nationales Forschungsprogramm 48 "Landschaften und Lebensräume der Alpen" des Schweizerischen Nationalfonds; [Synthesebericht NFP 48], vdf, Hochsch.-Verl. an der ETH, Zürich, 2007.

Backhaus, N., Reichler, C., und Stremlow, M.: Conceptualizing Landscape: An Evidence-based Model with Political Implications, Mt. Res. Dev., 28, 132-139, 2008.

Backhaus, N. und Stremlow, M.: Handlungsraum Landschaft: Wege zur Förderung transdisziplinärer Zusammenarbeit, Natur und Landschaft, 85, 345-349, 2010.

Banks, M.: Using Visual Data In Qualitative Research, 1. Aufl., SAGE Publications Ltd., London, 2007.

Bätzing, W.: Die Alpen: Geschichte und Zukunft einer europäischen Kulturlandschaft, 2. Aufl., Beck, München, 2003.

Bätzing, W.: Orte guten Lebens: Die Alpen jenseits von Übernutzung und Idyll, Rotpunktverlag, Zürich, 2009.

Benz, R., Dumermuth, M., Hein, S., Kaufmann, S., Krüsi, H., und Lugon, A.: ÖQV- Vernetzungsprojekte erfolgreich umsetzten, Fallbeispiele aus der Praxis, 2009.

Bourassa, S. C.: The aesthetics of landscape, Belhaven Press, London, New York, 1991.

Bourdieu, P.: Entwurf einer Theorie der Praxis auf der ethnologischen Grundlage der kabylischen Gesellschaft, 1. Aufl., Suhrkamp, Frankfurt am Main, 1979.

Bourdieu, P.: Sozialer Sinn: Kritik der theoretischen Vernunft, 7. Aufl., Suhrkamp, Frankfurt am Main, 1993.

Bourdieu, P.: Ökonomisches Kapital - Kulturelles Kapital - Soziales Kapital, in: Die verborgenen Mechanismen der Macht, Herausgeber: Steinrücke, M., Schriften zu Politik \& Kultur, 1, VSAVerlag, Hamburg, 49-75, 2005.

Bundesamt für Umwelt: Landschaftskonzept Schweiz: Teil I KONZEPT; Teil II BERICHT, Konzepte und Sachpläne, 2010.

Burckhardt, L., Ritter, M., und Schmitz, M.: Warum ist Landschaft schön?: Die Spaziergangswissenschaft, Schmitz, Kassel, 2006.

Burton, R. J. F.: Seeing Through the "Good Farmer's" Eyes: Towards Developing an Understanding of the Social Symbolic Value of "Productivist" Behaviour, Sociol. Ruralis, 44, 195-215, 2004.

Burton, R. J. F.: Understanding Farmers' Aesthetic Preference for Tidy Agricultural Landscapes: A Bourdieusian Perspective, Landscape Res., 37, 51-71, 2012.

Collier, J. und Collier, M.: Visual Anthropology: Photography as a research method, [Nachdr.], Univ. of New Mexico Press, Albuquerque, 2004.

Diener, R., Herzog, J., Meili, M., de Meuron, P., und Schmid, C.: Die Schweiz: Ein städtebauliches Portrait, Birkhäuser, Basel, 240-461, 2005.

Droz, Y. und Miéville-Ott, V.: Le paysage de l'anthropologue, in: La polyphonie du paysage, 1. Aufl., Herausgeber: Droz, Y., Miéville-Ott, V., und Chételat, J., Presses polytechniques et universitaires romandes, Lausanne, 5-20, 2005.

Droz, Y., Miéville-Ott, V., Spichiger, R., und Forney, J.: Le champ du paysage: Représentations paysagères et processus de légitimation des usages sociaux du paysage, De la Vue-des-Alpes au Pays-d'Enhaut, Neuchâtel, 2006.

Europarat: Europäisches Landschaftsübereinkommen, 2000.

European Cooperation in Science and Technology: COST 1007: Investigating Cultural Sustainability - Cultural sustainability, http: //www.culturalsustainability.eu/, letzter Zugriff: 4. April 2013.

Ewald, K. C. und Klaus, G.: Die ausgewechselte Landschaft: Vom Umgang der Schweiz mit ihrer wichtigsten natürlichen 
Ressource, 1. Aufl., Haupt, Bern, Stuttgart, Wien, 2009.

Flick, U.: Qualitative Sozialforschung: Eine Einführung, Originalausg., vollständig überarb. und erw. Neuausg., Rowohlt Taschenbuch Verlag, Reinbek, 2007.

Frey, R. L.: Starke Zentren-Starke Alpen: Wie sich die Städte und ländlichen Räume der Schweiz entwickeln können, Verlag Neue Zürcher Zeitung, Zürich, 2008.

Gailing, L. und Leibenath, M.: Von der Schwierigkeit, „Landschaft“ oder „Kulturlandschaft" allgemeingültig zu definieren, Raumforschung und Raumordnung, 1-12, 2011.

Garrod, B.: Exploring place perception a photo-based analysis, Ann. Tourism Res., 35, 381-401, 2008.

Gee, K.: Landschaftswandel und Wahrnehmung - Das Beispiel Offshore-Windkraft, in: Kulturlandschaft verstehen, Herausgeber: Reeh, T., Ströhlein, G., und Bader, A., Göttinger Schriften zu Landschaftsinterpretation und Tourismus, 5, Universitätsdrucke Göttingen, Göttingen, 85-102, 2010.

Heiland, S.: Zwischen Wandel und Bewahrung, zwischen Sein und Sollen: Kulturlandschaft als Thema und Schutzgut in Naturschutz und Landschaftsplanung, in: Kulturlandschaften als Herausforderung für die Raumplanung: Verständnisse - Erfahrungen - Perspektiven, Herausgeber: Matthiesen, U., Danielzyk, R., Heiland, S., und Tzschaschel, S., Verl. der ARL, Hannover, 43-70, 2006.

Hokema, D.: Die Landschaft der Regionalentwicklung: Wie flexibel ist der Landschaftsbegriff?, Raumforschung und Raumordnung, 239-249, 2009.

Hunziker, M.: Einstellungen der Bevölkerung zu möglichen Landschaftsentwicklungen in den Alpen, Bibliothek WSL, Birmensdorf, 2000.

Hunziker, M.: Wahrnehmung und Beurteilung von Landschaftsqualitäten - ein Literaturüberblick, in: Landschaftsqualitäten: Festschrift für Prof. Dr. Klaus C. Ewald anlässlich seiner Emeritierung im Jahr 2006, Herausgeber: Tanner, K. M., Bürgi, M., und Coch, T., Haupt, Bern, Stuttgart, Wien, 39-55, 2006.

Hunziker, M., Gehring, K., Kianicka, S., und Buchecker, M.: Wer will welche Landschaft?, TEC21, 45, 18-22, 2007.

Ipsen, D.: Ort und Landschaft, 1. Aufl., VS Verlag für Sozialwissenschaften, Wiesbaden, 2006.

Junge, X., Hunziker, M., und Schüpbach, B.: Indikatoren für Landschaftsqualität im Sömmerungsgebiet: Schlussbericht, Zürich, Birmensdorf, 30, 2010.

Junge, X., Lindemann-Matthies, P., Hunziker, M., und Schüpbach, B.: Aesthetic preferences of non-farmers and farmers for different land-use types and proportions of ecological compensation areas in the Swiss lowlands, Biol. Conserv., 144, 1430-1440, 2011.

Kaplan, R. und Kaplan, S.: The experience of nature: A psychological perspective, Cambridge University Press, Cambridge, New York, 1989.

Knox, P. L., Marston, S. A., und Gebhardt, H.: Humangeographie, 4. Aufl., Spektrum, Heidelberg, 791 S., 2008

Krysmanski, R.: Die Nützlichkeit der Landschaft: (1971), in: Landschaftswahrnehmung und Landschaftserfahrung: Texte zur Konstitution und Rezeption von Natur als Landschaft, Herausgeber: Gröning, G. und Herlyn, U., Minerva Publikation, München, 131-151, 1990.

Kühne, O.: Distinktion, Macht, Landschaft: Zur sozialen Definition von Landschaft, 1. Aufl., VS, Verl. für Sozialwiss., Wiesbaden,
2008.

Kühne, O.: Grundzüge einer konstruktivistischen Landschaftstheorie und ihre Konsequenzen für die räumliche Planung, Raumforschung und Raumordnung, 67, 395-404, 2009.

Lamnek, S.: Qualitative Sozialforschung: Lehrbuch, 5. Aufl., Beltz, Weinheim, Basel, 2010.

Landwirtschaftsforum - UNESCO Biosphäre Entlebuch, http://www.biosphaere.ch/de.cfm/company/forums/ offer-GesellschaftUBE-Foren-323409.html, letzter Zugriff: 4. Februar 2013

Lauber, S.: Agrarstrukturwandel im Berggebiet: Ein agentenbasiertes, räumlich explizites Agrarstruktur- und Landnutzungsmodell für zwei Regionen Mittelbündens, ART, Ettenhausen, 2006.

Lehmann, B. und Steiger, U.: Interessenausgleich beim Produkt "Landschaft", in: Alpenwert: Landschaften und Lebensräume der Alpen. Themenheft III des NFP 48, Nationales Forschungsprogramm 48 "Landschaften und Lebensräume der Alpen" des Schweizerischen Nationalfonds (Hrsg.), 2006.

Lynch, K.: Das Bild der Stadt, 5. Aufl., Bertelsmann-Fachzeitschr., Gütersloh, 2007.

Mayring, P.: Qualitative Inhaltsanalyse, Forum: Qualitative Sozialforschung, 2000.

Mayring, P.: Qualitative Inhaltsanalyse: Grundlagen und Techniken, 11. Aufl., Beltz, Weinheim, 2010.

Meier Ziegler, R. und Ziegler, F.: Das Meiental: http://www. meiental.ch/de/Meiental.html, letzter Zugriff: 4. Februar 2013.

Meynen, E. und Schmithüsen, J.: Handbuch der naturräumlichen Gliederung Deutschlands: Erste Lieferung, Verlag der Bundesanstalt für Landeskunde, Bad Godesberg, 1953.

Miéville-Ott, V.: Les représentations paysannes de la nature et du paysage, in: On achève bien les paysans: Reconstruire une identité paysanne dans un monde incertain, Herausgeber: Droz, Y. und Miéville-Ott, V., Georg Ed, Chêne-Bourg, 59-101, 2001.

Miéville-Ott, V. und Droz. Y.: Évolution de la réflexion paysagère en Suisse. A partir du programme Paysages et habitats de l'arc alpin, Économie rurale, 46-59, 2010.

Nassauer, J. I. und Westmacoot, R.: Progressiveness Among Farmers as a Factor in Heterogeneity of Farmed Landscapes, Ecological Studies, 64, 199-210, 1987.

Niederer, A. und Anderegg, K.: Alpine Alltagskultur zwischen Beharrung und Wandel: Ausgewählte Arbeiten aus den Jahren 1956 bis 1991, 2. Aufl., Haupt, Bern, Stuttgart, Wien, 1996.

Norer, R.: Lebendiges Agrarrecht: Entwicklungslinien und Perspektiven des Rechts im ländlichen Raum, Springer, Wien, 2005.

Pauwels, L.: Visual Sociology Reframed: An Analytical Synthesis and Discussion of Visual Methods in Social and Cultural Research, Sociological Method. Res., 38, 545-581, 2010.

Pinto-Correia, T., Gustavsson, R., und Pirnat, J.: Bridging the Gap between Centrally Defined Policies and Local Decisions - Towards more Sensitive and Creative Rural Landscape Management, Landscape Ecol., 21, 333-346, 2006.

Prosser, J. und Schwartz, D.: Photography within the Sociological Research Process, in: Image-based research: A sourcebook for qualitative researchers, Herausgeber: Prosser, J., Falmer Press, London, 115-130, 1998.

Ritter, J.: Landschaft. Zur Funktion des Ästhetischen in der modernen Gesellschaft. (1978), in: Landschaftswahrnehmung und Landschaftserfahrung: Texte zur Konstitution und Rezeption von Natur als Landschaft, Herausgeber: Gröning, G. und Herlyn, U., 
Minerva Publikation, München, 23-41, 1990.

Schallberger, P.: Wovon handeln bäuerliche Zukunftsvorstellungen?: Determinanten, Dimensionen und Typen, 1999.

Schenk, A., Hunziker, M., und Kienast, F.: Factors influencing the acceptance of nature conservation measures - A qualitative study in Switzerland, J. Environ. Manage., 83, 66-79, 2007.

Schmidt, E.: Der Landwirt und sein Verhältnis zur Landschaft, Diplomarbeit, Architektur Stadtplanung Landschaftsplanung, Universität Kassel, Kassel, 2005.

Schmidt, G.: Wanderung mit Bauern: Oder: Über das Schöne und Nützliche in modernen Kulturlandschaften, in: Kritischer Agrarbericht 2009: Landwirtschaft im Klimawandel, Herausgeber: Stodiek, F., ABL Bauernblatt Verl.-Ges., Hamm, Westfalen, 266-270, 2009.

Schüpbach, B., Briegel, R., Junge, X., Lindemann-Matthies, P., und Walter, T.: Ästhetische Bewertung landwirtschaftlicher Kulturen durch die Bevölkerung, ART, Ettenhausen, 2009.

Setten, G.: The habitus, the rule and the moral landscape, Cult. Geogr., 11, 389-415, 2004.

Steffen-Odermatt, S.: Mehr Lebensfreude dank mehr „Unordnung“: Schüpfheim: Öko-Vernetzung geht in die zweite sechsjährige Phase, Entlebucher Anzeiger, 5. Oktober 2012, 13, 2012.

Stotten, R., Rudmann, C., und Schader, C.: Rollenverständnis von Landwirten: Produzenten oder Landschaftspfleger?, in: Selbst- und Fremdwahrnehmung der Landwirtschaft, Herausgeber: Helmle, S., Margraf, Weikersheim, 41-51, 2010.
Sutherland, L.-A. und Burton, R. J.: Good Farmers, Good Neighbours? The Role of Cultural Capital in Social Capital Development in a Scottish Farming Community, Sociol. Ruralis, 51, 238255, 2011.

Tessin, W.: Ästhetik des Angenehmen: Städtische Freiräume zwischen professioneller Ästhetik und Laiengeschmack, 1. Aufl., VS Verlag für Sozialwissenschaften, Wiesbaden, 2008.

Tress, B. und Tress, G.: Begriff, Theorie und System der Landschaft: Ein transdisziplinärer Ansatz zur Landschaftsforschung, Naturschutz und Landschaftsplanung, 33, 52-58, 2001.

Umbricht, M. J.: Welche Landschaft wollen wir?: Denkmodelle für die Landschaft der Zukunft, Dissertation, Naturwissenschaften, Eidgenössische Technische Hochschule, Zürich, 2003.

Weiss, H.: Was heisst Qualität der Landschaft?: Gedanken über Wahrnehmung, Nichtwahrnehmung und die objektive Subjektivität des Landschaftsbegriffs, in: Landschaftsqualitäten: Festschrift für Prof. Dr. Klaus C. Ewald anlässlich seiner Emeritierung im Jahr 2006, Herausgeber: Tanner, K. M., Bürgi, M., und Coch, T., Haupt, Bern, Stuttgart, Wien, 15-22, 2006.

Wilson, G. A.: From productivism to post-productivism... and back again? Exploring the (un)changed natural and mental landscapes of European agriculture, T. I. Brit. Geogr., 26, 77-102, 2001.

Wöbse, H. H.: Landschaftsästhetik: Über das Wesen, die Bedeutung und den Umgang mit landschaftlicher Schönheit, Ulmer, Stuttgart, 2002. 